\title{
Risk Evaluation of Gas Pipeline Based on Rough Set
}

\author{
Gu Hongyan \\ Hebei College of Industry and Technology, Shijiazhuang 050091 \\ guohongyanguo@163.com
}

Keywords: gas pipeline; accident loss; rough set

\begin{abstract}
Gas is a flammable, explosive and high-pressure medium. Gas pipelines are passing through many different geographical conditions of changing natural environment with gas. Therefore, a lot of adverse consequences will be bought by the gas pipeline failure, however not all consequences have a great impact on assessment of the losses. The rough set theory is used to simplify the accident loss. In this paper, the accident loss of gas pipelines of the gas enterprise in one city taken as an example is evaluated and forecasted with the rough set theory, which can bring the scientific foundation for the manager's management and decision. Above 90 percent of city gas pipelines are underground, and they drill through some broad districts where terrain is complex and soil quality is very different. Occurrences of accidents do not take on obvious because of inflammable, explosive and high-pressure medium. The great trouble is brought in the latter part of the risk assessment due to many influencing factors. Exporting problems in decision-making or classification rules, and removing redundancy factors through knowledge (attributes) reduction with the rough set theory in this article.
\end{abstract}

\section{Pipeline Segments}

The difference of the risk evaluation of the pipeline and other devices is that there is not the same risk of sexual orientation on the entire pipeline. The pipeline is divided into different pipe sections that were evaluated by some indicator because complex combination of a variety of conditions in the pipeline and the entire pipeline risks in varying degrees. The pipeline should be divided according to population density, soil conditions, geological conditions, the situation of anticorrosion layer, piping useful life, a staging point should be inserted when the above-mentioned characteristics change significantly. A pipeline by more number of segments, the more accurate the results of the evaluation of each pipe section, but the higher costs may be caused in the data acquisition, processing and maintenance. On the other hand, if the division section of pipe is longer, the cost of data acquisition may be reduced, but the accuracy of evaluation may be reduced. The location of the regulator station in the built medium pressure pipeline is more obvious, and some medium pressure pipeline crossing the railway or railway accompanied, in some places the population density is high, in some places road construction is more intensive, therefore the regulator station as a staging point in the medium pressure of the pipeline is divided into 7 segments, both the important changes of the pipeline can be reflected, but also the staging point of the pipeline is more obvious, and good principles consistent with the pipeline segment. Figure 1 shows the pipeline segments later:

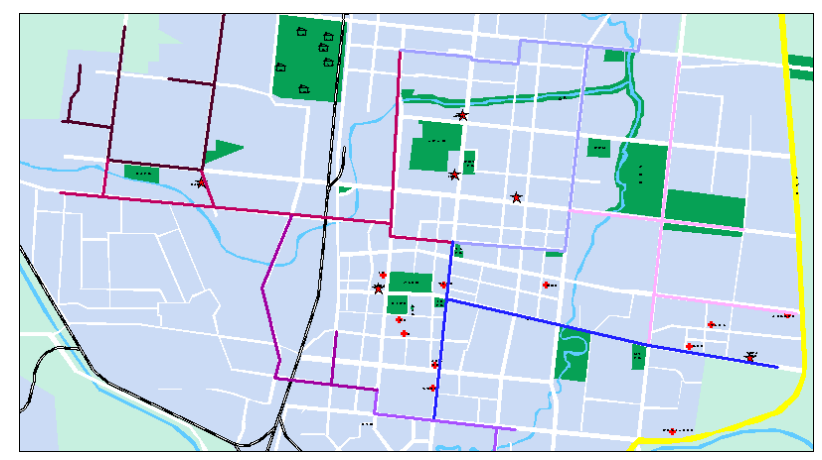

Fig. 1. The subsection of the pipeline 


\section{Data Preparation}

Once the pipeline failure, many adverse consequences will appear. Such as casualties, environm ental pollution and property damage caused by the failure, the impact of the gas supply system, the 1 evel of maintenance, maintenance costs, repair time, the social impact of failure, the people spiritual and mental trauma and so on.

Casualties caused by failure

The casualties include the number of deaths, the number of injuries and the number of injured $\mathrm{m}$ aimed.

Impact on the environment

Liquefied gas is a flammable, explosive dangerous goods, leakage may occur In the process of tr ansportation, storage and use, explode will occur when the leak larger number reached liquefied exp losive limit in the air or the case of fire, causing serious damage and environmental impact to the su rrounding environment and the production and living facilities.

Property damage caused by failure

Economic loss is divided into the direct economic losses and indirect economic losses. The direct economic loss is the sum of economic losses including the native accident disaster and secondary $\mathrm{d}$ isasters closely. Indirect economic losses is that the pause and disorders of the enterprise and social and management, and the potential environmental damage is caused by accident.

The impaction of the gas supply system.

Once the pipeline failure, discontinued and maintenance will occur in air supply system.

The level of maintenance

Maintenance costs

The time to repair

The social impact caused by the failure

The social impact caused by the failure is the qualitative description, including stagnation of city life, destruction of urban rhythm, destruction of the city's image.

The people spiritual and mental trauma caused by failure

Mental and psychological trauma is that accident disaster shadows to the people caused panic, fe ar and frightened.

\section{Structural Information Table}

Some factors in the evaluation of consequences of the loss of pipeline accidents can be described with specific numbers, but many factor can be descripted with logic language not with specific nu merical, such as: the mental trauma caused by failure to the people can be divided into no impact, $g$ eneral impact, great impact causing panic. The specific indicators grading are shown in Table 1:

According to the buried pipeline, the pipeline across the entire city, and the factors involved in th e daily life of the city's residents, the capacity of ordinary people, the determine level of each factor is shown in Table 2.

When giving the following data, the uncertainty integrity and simplicity of the data must be en sured. Uncertainty is that the data noise must be less. Knowledge discovery from data containing no ise will affect the credibility of the knowledge. Data noise will mask the true knowledge, and may $i$ nfluence the credibility of the final decision-making rules. Integrity embodied in two aspects: First, the value of the property is not a null value, the second is whether the required data is comprehensiv e. Easier to achieve the first and the second need experts to grasp. Simplicity is to try to select the $\mathrm{i}$ mportant attributes, eliminate redundancy. In decision-making, decision-makers tend to seize the $\mathrm{m}$ ain factors of the problem, rather than the details of the problem was very clear. In mining rules, the more the number of the feature, the greater the chance of noise generated. Therefore select a small er set of typical characteristics, not only conforms to the psychology of decision-makers, and easy a ccess to concise knowledge, to reduce the dimension of the knowledge of the state space by attribut e reduction. 
Table 1 . The grade division of every factor of fault result

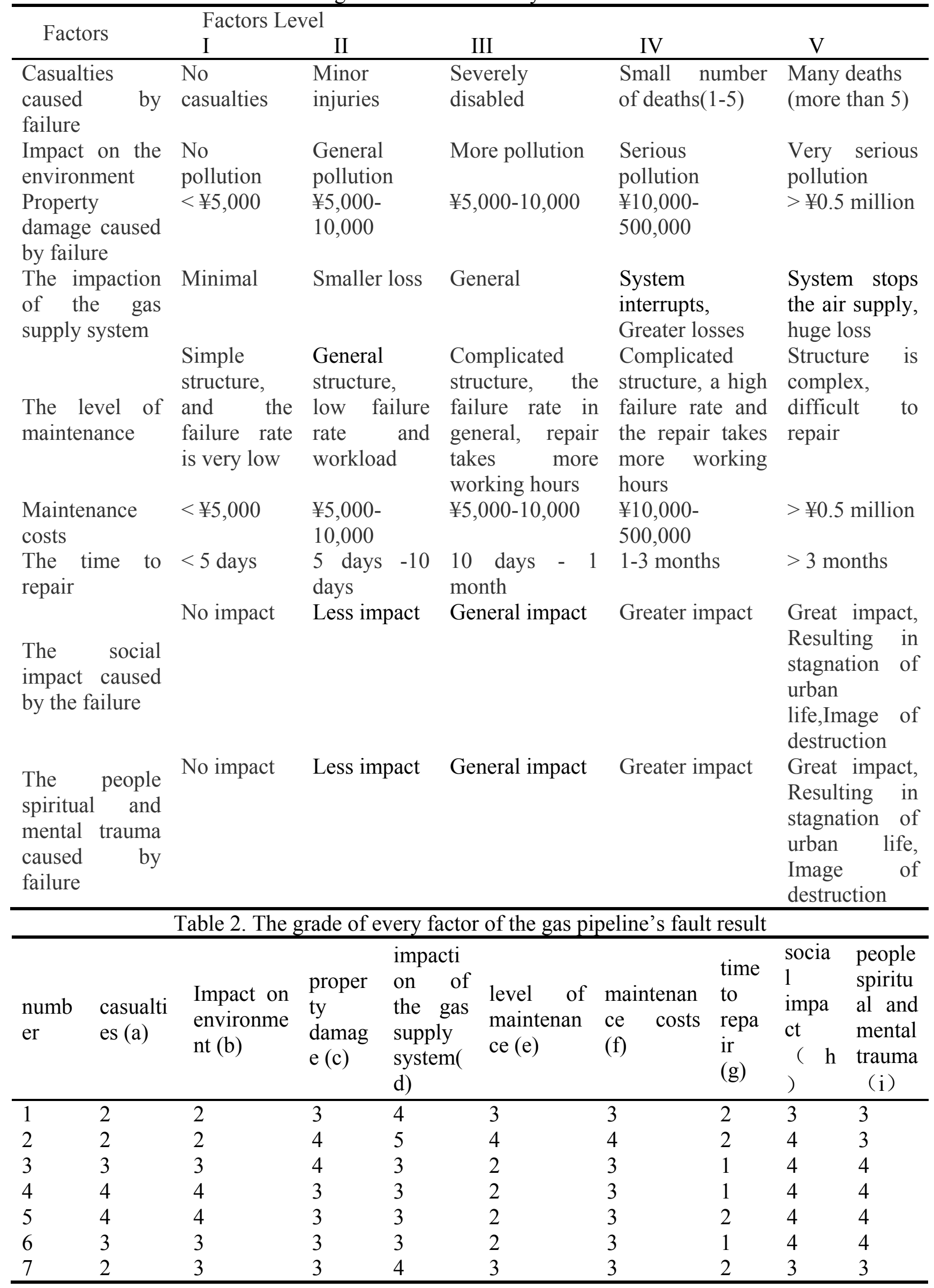




\section{The Attribute Reduction of Information Table}

Attribute reduction is two steps: the first step is to find nuclear properties, the second step is to fi nd the smallest reduction. the condition attributes are removed one by one by the data analysis meth od, To determine whether the correct classification of impact, impact compared to the nuclear prope rties, or for non-nuclear properties. $U$ is the universe of discourse, $R$ is the equivalence relation on U.

$\{\{1\},\{2\},\{3\},\{4\},\{5\},\{6\},\{7\},\{8\}\}$

$=\{\{1\},\{2\},\{3,6\},\{4\},\{5\},\{7\},\{8\}\}$

So a is nuclear properties;

$=\{\{1,7\},\{2\},\{3\},\{4\},\{5\},\{6\},\{8\}\}$

So $\mathrm{b}$ is nuclear properties;

$=\{\{1,2\},\{3\},\{4\},\{5\},\{6\},\{7\},\{8\}\}$

So c is nuclear properties;

$=\{\{1\},\{2\},\{3\},\{4\},\{5\},\{6\},\{7\},\{8\}\}$

So $\mathrm{d}$ is remove attributes;

$=\{\{1\},\{2\},\{3\},\{4\},\{5\},\{6\},\{7\},\{8\}\}$

So e is remove attributes;

$=\{\{1\},\{2\},\{3\},\{4,5\},\{6\},\{7\},\{8\}\}$

So e is remove attributes;

$=\{\{1\},\{2\},\{3\},\{4,5\},\{6\},\{7\},\{8\}\}$

So $\mathrm{g}$ is nuclear properties;

$=\{\{1\},\{2\},\{3\},\{4,6\},\{5\},\{7\},\{8\}\}$

So $\mathrm{h}$ is nuclear properties;

$=\{\{1\},\{2\},\{3\},\{4\},\{5\},\{6\},\{7\},\{8\}\}$

So $\mathrm{i}$ is remove attributes;

From the above discussion, the core attributes is $=\{\mathrm{a}, \mathrm{b}, \mathrm{c}, \mathrm{g}, \mathrm{h}\}$.

Upon examination that:

So, $\{\mathrm{a}, \mathrm{b}, \mathrm{c}, \mathrm{g}, \mathrm{h}\}$ is the only reduction.

Removing redundant attributes $\mathrm{d}, \mathrm{e}, \mathrm{f}$ and $\mathrm{i}$ is shown in Table 3:

Table 3. The information table of deleting redundancy attribute

\begin{tabular}{lllllll}
\hline number & $\begin{array}{l}\text { casualties } \\
\text { (a) }\end{array}$ & $\begin{array}{l}\text { Impact } \\
\text { environment (b) }\end{array}$ & $\begin{array}{l}\text { on } \\
\text { damage }(\mathrm{c})\end{array}$ & $\begin{array}{l}\text { time to repair } \\
(\mathrm{g})\end{array}$ & $\begin{array}{l}\text { social } \\
(\mathrm{h})\end{array}$ & impact \\
\hline 1 & 2 & 2 & 3 & 2 & 3 \\
2 & 2 & 2 & 4 & 2 & 4 \\
3 & 3 & 3 & 4 & 1 & 4 \\
4 & 4 & 4 & 3 & 1 & 4 \\
5 & 4 & 4 & 3 & 2 & 4 \\
6 & 3 & 3 & 3 & 1 & 4 \\
7 & 2 & 3 & 3 & 2 & 3 \\
\hline
\end{tabular}

\section{References}

[1]Li Xiaoxia, Chen Mianyun. Simplify of condition attributes of decision tables in rough set theory. Huazhong University of Science and Technology University (Natural Science Edition), vol. 31(8):85-87(2003). 
[2]An Liping, Chen Zengqiang. multi-attribute decision analysis based on rough set theory. Journal of Management Sciences. vol. 5(5):21-25(2003).

[3]Wu Fubao, Li Qi. Inductive learning method based on a knowledge representation system in rough set theory. Control and decision-making, vol. 14(3):206-211(1999).

[4]Mrozek A. Rough sets and dependency analysis among attributes in computer implementations of experts inference models. Int. Jour. Man-Machine Studies, vol. 30(4):457-473(1989).

[5]Han Bin, Wu Tiejun. The dynamic attribute reduction combined with rough set theory. Systems engineering theory and practice,6:67-73(2002).

[6]Dai Lianshuang, Zhang Junyi, Zhang Xin, et al. RiskScore Pipeline Risk Assessment Method and Its Application. Oil \& Gas Storage and Transportation. Vol, 29(11):818-820(2010).

[7]Zhang Xian-yong, Xiong Fang, Mo Zhiwen, et al. Rough Set Model of Logical Difference Operation of Grade and Precision. Journal of University of Electronic Science and Technology of China, Vol. 39(5):783-787(2010).

[8]Zhao Lian-sheng,Shi Ji-hua. Real Value Attribute Reduction Method Based on Rough Sets. Journal of Inner Mongolia University, vol. 41(1):97-101(2010).

[9]Fan Chi-jie, Chen Limin, Xia Chunyan. The Approach for Attributes Reduction Based on Rough Set Theory. Microcomputer information, vol. 26(2):222-228(2010).

[10]Teng Shuhu, Zhou Shilin, Sun Jixiang, et al. Attribute Reduction Algorithm Based on Conditional Entropy under Incomplete Information System. Journal of National University of Defense Technology, vol. 21(1):90-94(2010). 